\title{
EchoGéo
}

\section{Le nouveau Xinjiang : intégration et recompositions territoriales d'une périphérie chinoise}

\section{Alain Cariou}

\section{OpenEdition}

\section{Journals}

Édition électronique

URL : https://journals.openedition.org/echogeo/11244

DOI : 10.4000/echogeo.11244

ISSN : 1963-1197

Éditeur

Pôle de recherche pour l'organisation et la diffusion de l'information géographique (CNRS UMR 8586)

Référence électronique

Alain Cariou, « Le nouveau Xinjiang : intégration et recompositions territoriales d'une périphérie chinoise », EchoGéo [En ligne], 9 | 2009, mis en ligne le 17 juin 2009, consulté le 01 août 2021. URL http://journals.openedition.org/echogeo/11244 ; DOI : https://doi.org/10.4000/echogeo.11244

Ce document a été généré automatiquement le 1 août 2021

EchoGéo est mis à disposition selon les termes de la licence Creative Commons Attribution - Pas d'Utilisation Commerciale - Pas de Modification 4.0 International (CC BY-NC-ND) 


\title{
Le nouveau Xinjiang : intégration et recompositions territoriales d'une périphérie chinoise
}

\author{
Alain Cariou
}

1 Il est de coutume de présenter le Xinjiang, vaste province chinoise la plus à l'Ouest du pays, comme une périphérie continentale, marge culturelle du territoire chinois en proie au sous-développement et au séparatisme. Constituant la partie orientale de l'Asie centrale, le Xinjiang, ex-Turkestan oriental, se rattache en effet culturellement au monde turco-mongol et bénéficie au regard de l'importance de ses minorités nationales du statut de région autonome depuis 1955 (fig. 1). A cette marginalité culturelle, l'analyse régionale classique ajoute la marginalité géographique de cette vaste région confrontée à des conditions naturelles souvent qualifiées d'extrêmes. Couvrant 1,6 million de $\mathrm{km}^{2}$ (1/6 du territoire chinois) pour 20,5 millions d'habitants, le Xinjiang est caractérisé par l'immensité des dépressions désertiques et steppiques ceinturées par de hautes chaînes de montagnes dont les sommets dépassent parfois les 7000 mètres d'altitude. Ainsi cette Chine intérieure apparait à bien des égards comme une autre Chine, Chine occidentale du vide (densité de $13 \mathrm{hab} . / \mathrm{km}^{2}$ ), de l'enclavement, sorte de cul-de-sac et de réduit d'archaïsme ${ }^{1}$ en contre-point d'une Chine orientale ouverte tournée vers la modernité et la mondialisation. Cette perception géographique découle en partie du contexte historique récent durant lequel les confins sinosoviétiques étaient militarisés et hermétiquement fermés en raison de la brouille opposant les deux puissances communistes si bien qu'on a pu qualifier cette Asie centrale d' " hinterland amorphe » (Aubin, 1990). 
Fig. 1 : LE XINJIANG ENTRE CHINE ET ASIE CENTRALE

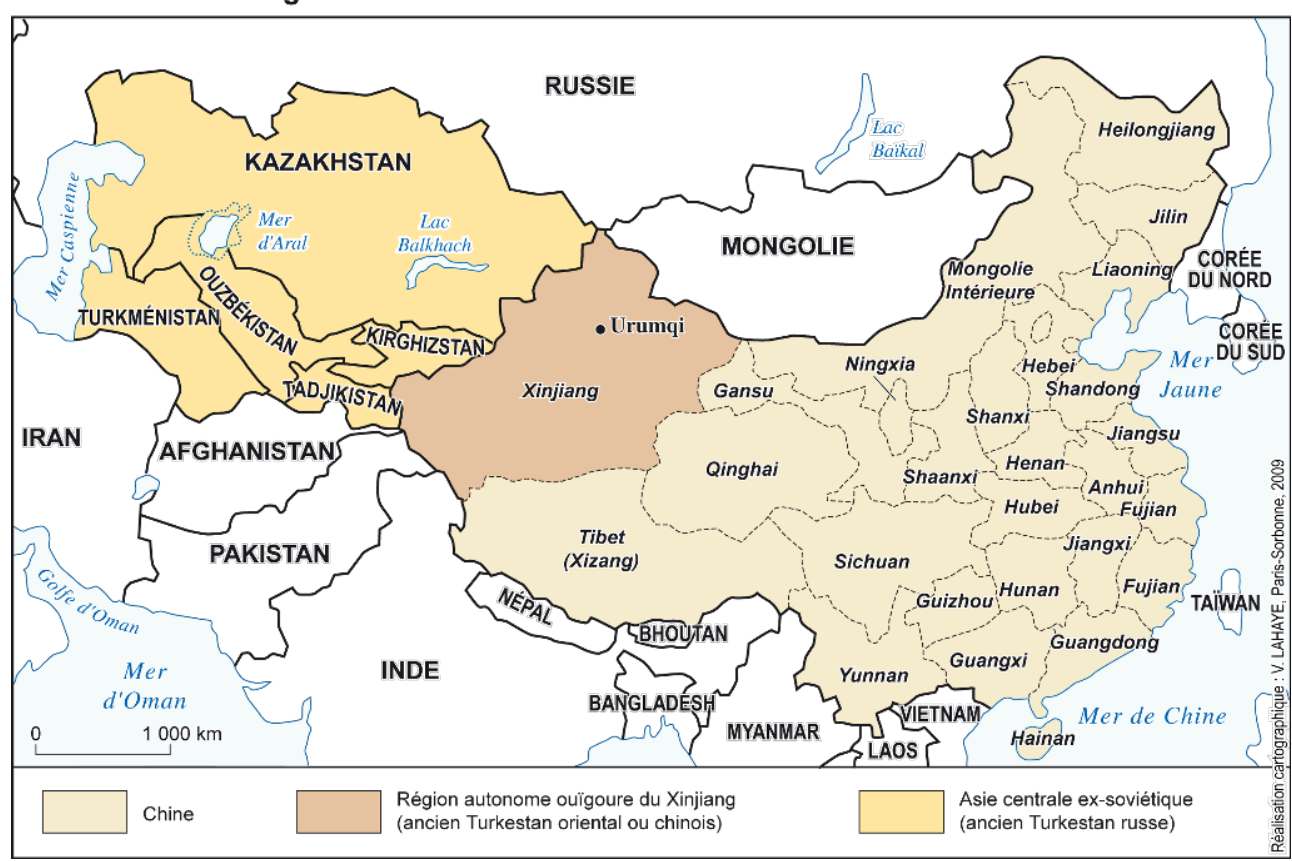

2 Si ce statut de périphérie conféré au Xinjiang n'est pas à nier, il doit cependant être nuancé, surtout au regard des bouleversements qui l'affectent depuis une vingtaine d'années. En effet, cette région frontalière présente aujourd'hui le paradoxe d'un développement accéléré qui la singularise des autres provinces intérieures de la Chine par son premier rand pour son IDH et son PIB mais également par son attraction qui se caractérise par un solde migratoire positif (Pannell et Schmidt, 2006).

Il convient alors de s'interroger sur les raisons de ce développement atypique dans le cadre de l'Ouest chinois et de montrer combien les stratégies de développement en cours sont porteuses de recompositions socio-territoriales. Il faut partir d'une analyse géostratégique pour comprendre pourquoi cet espace bénéficie de la part du pouvoir central d'une stratégie de développement prioritaire qui l'érige en vaste front pionnier. Si le discours officiel insiste sur les bienfaits économiques apportés au Xinjiang par une valorisation des ressources naturelles il reste en revanche très discret sur l'autre finalité du projet qui vise sa sinisation. La stratégie à l'œuvre consiste à parachever l'intégration économique et culturelle d'une périphérie jugée turbulente et de la faire «rentrer» dans la logique de l'Etat-nation. Cependant les enjeux de la région ne se limitent pas uniquement au cadre national, mais s'inscrivent désormais à l'échelle eurasiatique. Depuis la disparition de l'URSS, la Chine se tourne résolument vers l'Asie centrale ex-soviétique où elle s'impose de plus en plus comme un acteur économique et politique incontournable. Ainsi, d'une situation géographique de marge enclavée et fermée, le Xinjiang se mue progressivement en interface active, offrant le visage d'une nouvelle fenêtre continentale ouverture sur l'Eurasie, terre d'expansion de l'influence chinoise. 


\section{Le Xinjiang : vers une intégration à l'Etat-nation chinois}

\section{Une nouvelle frontière à conquérir}

4 Jamais la notion de Xinjiang, ou littéralement «nouvelle frontière » en chinois mandarin, n'a été autant d'actualité. Cette toponymie appliquée aux terres centrasiatiques lors de leur conquête au XVIII ${ }^{\mathrm{e}}$ siècle par la dynastie des Qing prend aujourd'hui tout son sens. Il s'agit ici d'entendre par frontière, un " espace-frontière " faiblement maîtrisé où s'exerce une politique étatique volontariste de valorisation territoriale afin d'occuper, de contrôler et d'intégrer une région frontalière parfois contestée par les minorités nationales (Renard et Picouet, 1993). L'actuelle situation du Xinjiang illustre bien ce concept de frontière, dans le sens où, à l'image de l'Ouest américain au XIX ${ }^{e}$ et de l'Asie centrale russe puis soviétique, les flux de migrants han ${ }^{2}$ investissent et organisent progressivement l'ouest de cet immense territoire de la République populaire de Chine traditionnellement occupé par des populations locales non-chinoises (fig. 2).

Fig. 2 : LE XINJIANG : INTÉGRATION, OUVERTURE ÉCONOMIQUE ET SINISATION

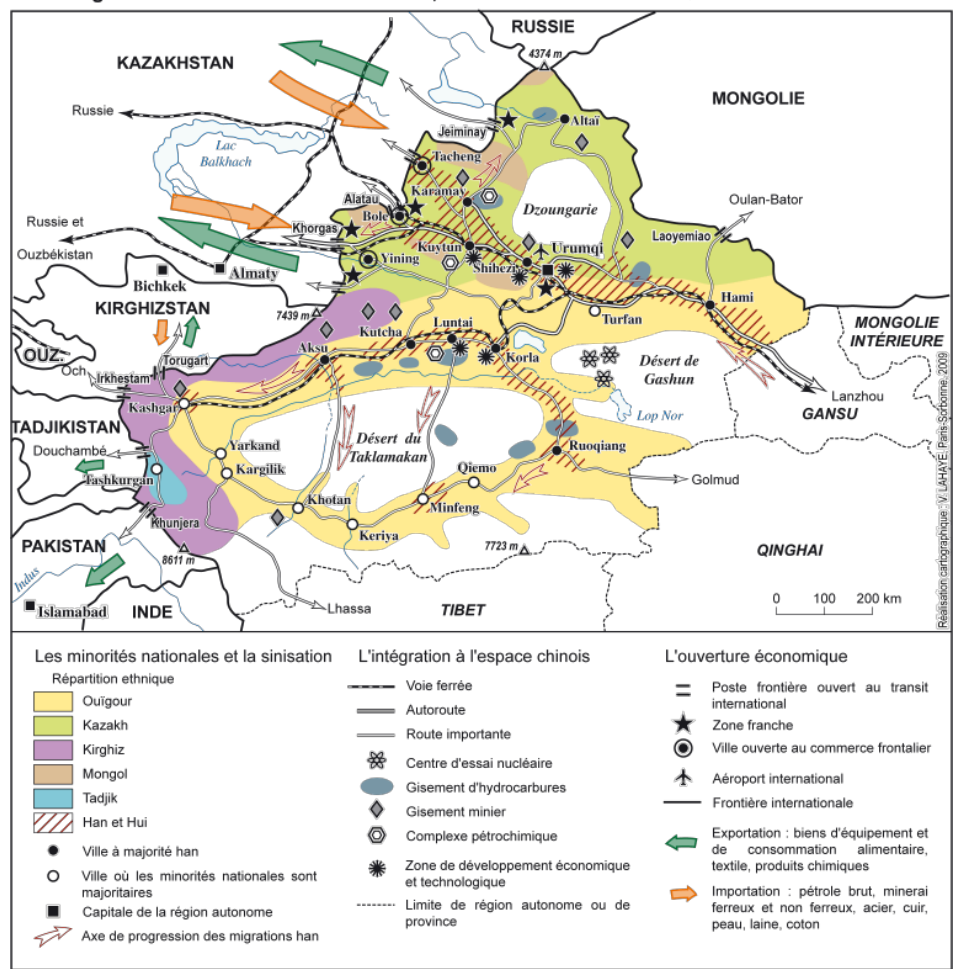

5 Si l'influence de la Chine dans la région n'est pas nouvelle en revanche son annexion par le pouvoir central est très récente car véritablement effective que depuis 1949. En effet, durant près de deux mille ans, la Chine a vainement tenté de porter les limites de son empire sur ces terres centrasiatiques mais sans jamais pouvoir s'y imposer durablement, refoulée à chaque fois par la poussée des peuples turcs et mongols. Motivée par le contrôle stratégique du commerce sur la Route de la soie, la colonisation chinoise s'infiltre dès l'Antiquité dans le bassin du Tarim sous la dynastie des Han (206 av. J.-C., 220 après J.-C.), reflux ensuite pour reprendre sous celle des Tang du VII au $\mathrm{IX}^{\mathrm{e}}$ siècles (Boulnois, 2001). Il faut ensuite attendre le XVIII ${ }^{\mathrm{e}}$ siècle pour que la Chine 
réoccupe la région puis la dote du statut de province en 1884 sans toutefois réussir à s'y imposer en maitre comme en témoignent les nombreux soulèvements des populations locales mais aussi l'influence et l'infiltration grandissantes des russes puis des soviétiques de la fin du $\mathrm{XIX}^{\mathrm{e}}$ au début du $\mathrm{XX}^{\mathrm{e}}$ siècle. Ainsi, par deux fois durant la première moitié $\mathrm{du} \mathrm{XX}^{\mathrm{e}}$ siècle, les éphémères républiques du Turkestan oriental (de 1933 à 1934 et de 1944 à 1949) viendront défier le pouvoir chinois (Wang, 1995). En définitive, la mainmise chinoise sur le Xinjiang n'a-t-elle été par le passé qu'intermittente, sans cesse remise en cause et contestée, c'est pourquoi, depuis la monté en puissance de la Chine nouvelle, Pékin n'entend plus se voir disputer sa souveraineté sur ces territoires de l'ouest.

6 Cette opiniâtreté à tenir l'espace s'explique par le rôle hautement stratégique que joue le Xinjiang à l'égard de la Chine. Si depuis l'Antiquité cette région est considérée par les Han comme une zone de colonisation où « la terre est abondante et la population peu nombreuse " les enjeux actuels portent surtout sur l'extraction des ressources naturelles dont l'économie chinoise en pleine croissance a tant besoin. Il s'agit d'intégrer une périphérie où les réserves de pétrole, de gaz naturel et de charbon représentent respectivement $30 \%, 34 \%$ et $40 \%$ des réserves estimées du pays (Xinhua, 2005). Corrélativement, le développement économique du Xinjiang doit aussi permettre de résoudre le problème géopolitique interne de la stabilité d'une marge-frontière en proie à un irrédentisme séculaire. En effet, au regard d'une histoire mouvementée où le pouvoir chinois dans la région a été mainte fois contesté par les populations autochtones mais aussi par le voisin russe, le Xinjiang est considéré par Pékin comme une zone sensible où plane toujours une double menace pour la sécurité de la Chine. A l'instar du Tibet voisin, la menace est avant tout intérieure, le séparatisme ouigour étant identifié par les autorités chinoises comme un sérieux facteur d'instabilité. Depuis les années 1960, l'intensification de la colonisation chinoise dans la région a en effet entraîné l'exaspération grandissante des minorités nationales. L'hégémonie des Han dans l'administration locale qui confine les élites locales à un rôle subalterne, l'attribution prioritaire de terres aux migrants han fraîchement arrivés des régions pauvres de l'est ou la politique de limitation des naissances dans des sociétés natalistes de tradition musulmane sont vécues quotidiennement comme autant de discriminations par les Ouïgours censées être autonomes. Si généralement l'attitude des populations autochtones à l'égard des Han se résume à un ressentiment hostile et fataliste, la situation de tension est parfois si forte qu'elle débouche sur la multiplication de manifestations souvent spontanées où sont lancés des slogans hostiles à la politique chinoise. Ces soulèvements dégénèrent parfois en émeute avec l'attaque de bâtiments administratifs et l'affrontement avec les forces de police. Afin de faire face au " problème oügour », le gouvernement inaugure en 1996 une politique de répression du « frapper fort » qui se traduit par l'arrestation de plus 18000 activistes et sympathisants de la cause ouïgoure (Lothaire, 2006). La mise en place d'un contrôle étroit de la population ainsi que la violence de la répression va engendrer la multiplication des mouvements nationalistes, dont le Parti de l'Islam du Turkestan Oriental, ainsi que la radicalisation des actions (Kumul, 1998). Les attentats à la bombe et les attaques contre la police, les cadres du Parti et les militaires émaillent désormais l'actualité du Xinjiang.

7 Outre ces problèmes intérieurs, pour Pékin, la menace est aussi extérieure surtout depuis 1991, date de l'implosion de l'URSS et de l'émergence corrélative des cinq nouvelles républiques centrasiatiques ${ }^{3}$ (Kellner, 2008). Ainsi, l'accession à 
l'indépendance des peuples turciques et persans d'ex-Union soviétique ne va pas sans poser de problème à la Chine. En effet, alors que ces derniers se sont émancipés de la tutelle soviétique, ceux du Xinjiang connaissent au contraire une intensification de la colonisation chinoise. Aussi, les autorités chinoises craignent que ce nouveau contexte géopolitique ne vienne éveiller les consciences et attiser les contestations et les revendications nationalistes chez les minorités nationales du Xinjiang car dans cette grande aire culturelle turco-iranienne, la proximité historique et culturelle est très grande entre les populations. Le problème devient d'autant plus épineux lorsque l'on sait l'extrême imbrication ethnique due notamment au caractère artificiel des frontières. Chaque ethnie titulaire d'une région ou d'un Etat est présente sous forme de minorité dans les territoires voisins. Outre leur région autonome où ils constituent $45,2 \%$ de la population en 2000 , les Ouïgour sont également présents sous forme de minorités au Kazakhstan ( $1 \%$ de la population), au Kirghizstan et en Ouzbékistan. Inversement il existe une grande communauté kazakhe au Xinjiang (plus de 1,2 million d'individus soit $6,74 \%$ de la population) tandis que la minorité kirghize et tadjike ne concerne que des effectifs beaucoup plus faibles (160000 Kirghiz et 40000 Tadjik au Xinjiang selon les chiffres officiels). Aux yeux de Pékin, le Xinjiang serait donc une boîte de Pandore, source permanente d'instabilité et d'insécurité sur sa frontière occidentale. La peur d'un nationalisme anti-chinois et d'une contamination par l'intégrisme musulman à partir de l'Afghanistan, du Pakistan, voire de l'Ouzbékistan explique l'empressement du gouvernement chinois à investir le terrain centrasiatique.

8 Au total, la multiplicité des enjeux géostratégiques concourent à faire du Xinjiang une des régions où les investissements et le développement sont considérés comme prioritaires. En effet, dans le cade du programme d'ouverture de l'Ouest de la Chine lancé en 2000 et qui concerne douze provinces ou régions autonomes, le Xinjiang se singularise comme l'entité la plus riche et la plus développée de ce Grand Ouest. Les objectifs affichés et les transformations en cours ambitionnent de parachever la conquête et l'intégration de cette "nouvelle frontière » à l'ensemble chinois tout en l'utilisant comme " poste avancé » afin de devenir une puissance continentale influente sur toute l'Eurasie.

\section{L'intégration par le désenclavement géographique}

9 La vulnérabilité de la souveraineté et de la colonisation chinoise au Xinjiang au cours des siècles tient en partie à la géographie. La distance et la difficulté des communications ont constituées jusqu'à très récemment un handicap à l'implantation han massive (Jan, 1998). Située à plus de $3500 \mathrm{~km}$ des provinces côtières, les garnisons militaires et les colonies de commerçants chinois implantées au Turkestan n'ont pu se maintenir durablement $\mathrm{du}$ fait de l'éloignement de leurs bases arrières. Ainsi, l'impérialisme han disputé au cours de l'histoire par l'expansionnisme nomades des peuples de Dzoungarie et par les populations oasiennes ouigour du bassin du Tarim a toujours achoppé dans ces contrées occidentales lointaines faute de liaison stable avec le reste de l'Empire du milieu. De plus, le monde centrasiatique aride des steppes, des déserts et des hautes montagnes peuplé de populations musulmanes non-chinoises à longtemps été perçu comme un territoire répulsif en raison de la distance culturelle et physique qui le sépare des grandes plaines agricoles orientales de la Chine. C'est pourquoi l'histoire du peuplement chinois au Xinjiang relève en grande partie de mesures coercitives sur ces terres de relégation. Le même processus se retrouve dans 
l'Asie centrale soviétique voisine où affluent koulaks, dissidents politiques et peuples punis par Staline.

En dépit de son éloignent du cœur politique, démographique et économique du pays, le Xinjiang est désormais désenclavé et les obstacles naturels ont été progressivement vaincus depuis à peine un demi siècle. La région autonome est en effet désormais arrimée au reste du pays grâce à l'aménagement de la route de l'ouest (Xiyu) qui depuis Lanzhou (Gansu) gagne Urumqi et le reste du Xinjiang. Cette route est en fait un axe multimodal où le rail et la route empruntent le corridor du Gansu, vaste couloir naturel qui, entre les hauts plateaux du Tibet et les déserts de Mongolie, fait communiquer les plaines orientales aux bassins de Dzoungarie et du Tarim. Le défi du désenclavement et de la pénétration chinoise au Xinjiang a en premier lieu reposé sur le chemin de fer, mode de transport particulièrement propice pour vaincre l'immensité continentale et permettre la circulation de convois massifs destinés à la conquête et à l'exploitation de l'Ouest. Avec la coopération du frère soviétique, la Chine aménage dans les années 1950 le chemin de fer du Nord-Xinjiang qui sur plus de $2350 \mathrm{~km}$ devait relier Lanzhou à la frontière sino-soviétique d'où une connexion avec le réseau soviétique était prévue. Suite au refroidissement des relations entre les deux géants communistes, la ligne stratégique Nord-Xinjiang va s'arrêter subitement à $200 \mathrm{~km}$ de la frontière et avoir pour terminus jusqu'en 1985 la ville de Usu située à l'ouest d'Urumqi. Pour faire face à la saturation de cette épine dorsale de la colonisation, le tronçon ferroviaire Lanzhou/ Urumqi est dédoublé sur $1622 \mathrm{~km}$ en 1995 faisant ainsi passer la capacité de transport de 11,5 millions de tonnes par an à 25 millions de tonnes. Outre le fret, c'est par cette ligne qu'afflue l'essentiel des migrants venus des provinces rurales pauvres de l'est du pays. Aujourd'hui un service régulier de trains de voyageurs permet de relier Pékin à Urumqi en $50 \mathrm{~h}$.

11 C'est à l'époque des tensions sino-soviétiques que le besoin d'un réseau routier moderne s'est fait sentir. Pour des raisons stratégiques, l'axe ferroviaire Lanzhou/ Urumqi est renforcé par une grande route stratégique qui sera ensuite complétée et ramifiée par des sections routières permettant la sécurisation des différentes frontières communes avec l'URSS, le Pakistan, l'Inde et la Mongolie. Avec l'apaisement des tensions internationales dans les années 1980 et l'essor de l'ouest chinois, le transport routier devient avec le rail un élément de la sinisation du Xinjiang. A partir de 1992, des travaux de modernisation sont engagés sur le grand axe routier Lanzhou/Urumqi si bien qu'aujourd'hui il est possible à partir de Lanzhou de gagner Khorgas sur la frontière kazakhe, via Hami et Urumqi, sans quitter l'autoroute (fig. 3). Enfin cet axe est depuis 2004 doté d'un gazoduc de $4200 \mathrm{~km}$ de long reliant les sites d'extraction de Luntai dans le nord du bassin du Tarim à Shanghai (Simonet, 2007). 
Figure 3 - L'autoroute Lanzhou/Urumqi

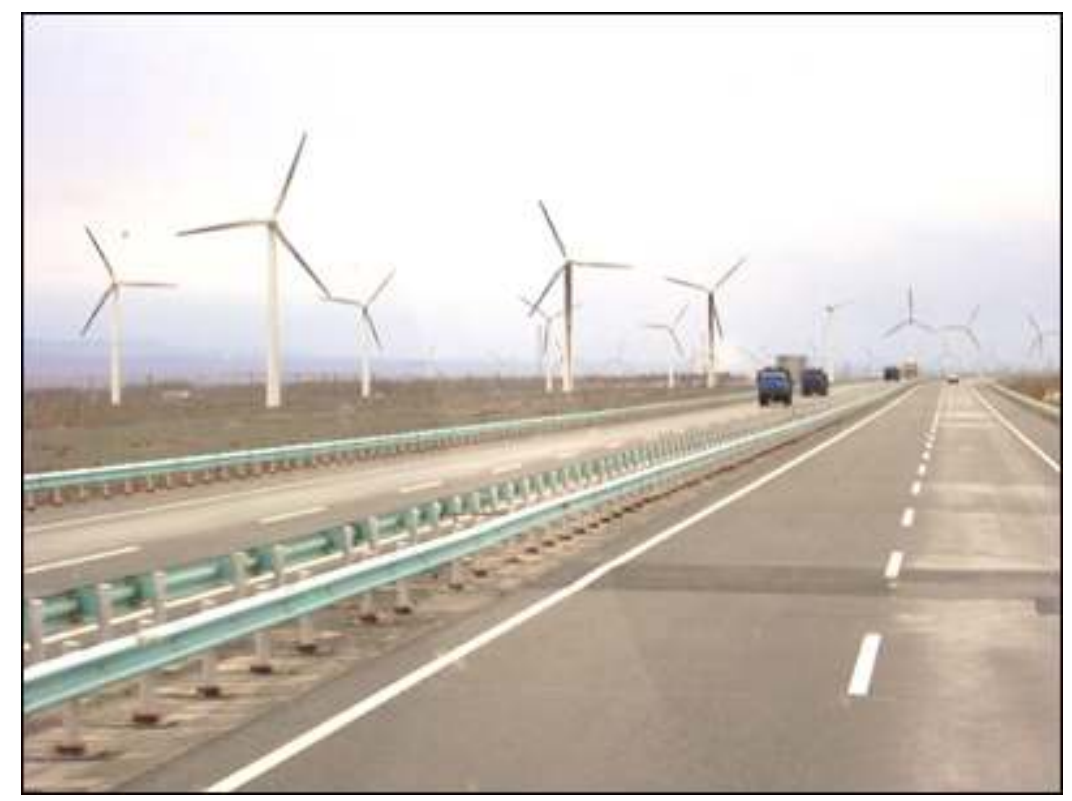

Cette autoroute est un axe majeur récent au service de l'intégration régionale (A. Cariou, 2008)

Outre le rôle de l'aéroport d'Urumqi relié à toutes les grandes villes chinoises, l'aménagement de ce corridor par des infrastructures modernes consacre l'intégration géographique du Xinjiang au reste du pays ce qui ouvre la voie à une assimilation économique et culturelle.

\section{L'intégration par la sinisation}

13 Si les peuples turco-mongols du Xinjiang ont le statut de minorité nationale à l'échelle du pays, ils sont aujourd'hui en passe d'être réduits au rang de minorités à l'échelle de leur propre région. A la fin du XIX ${ }^{\mathrm{e}}$ siècle les Han représentaient une partie infime de la population du Xinjiang, moins de 5000 familles, « un nombre qui n'atteignait même pas celui d'un canton en Chine intérieure » (Patterson Giersch, 2008). Ils constituaient officiellement $6,7 \%$ de population de la région autonome en 1949 mais 40,6\% en 2000. C'est donc à partir de la deuxième moitié $\mathrm{du} \mathrm{XX}^{\mathrm{e}}$ siècle que débute une véritable politique de transmigration han en direction du Xinjiang, le processus migratoire associée à la dynamique pionnière étant considéré par Pékin comme la solution au problème posé par "l'instabilité ethnique ». Grâce au désenclavement géographique, la toute nouvelle République populaire de Chine était à même de pouvoir transférer et installer massivement des colons d'est en ouest afin d'occuper et d'exploiter cette périphérie tout en protégeant ses frontières du voisin soviétique. A l'image des paysans-soldats installés dans l'Antiquité sur le limes de l'empire sous la dynastie des Han (Hopkirk, 1995), le pouvoir communiste instaure en 1954 une structure paramilitaire, le Corps de Production et de Construction du Xinjiang (plus communément appelé Bingtuan : la Brigade militaire), ayant pour mission de renforcer le contrôle politique du territoire par l'ouverture et l'organisation de fronts pionniers. Constitué au départ par les troupes démobilisées sur place de l'Armée populaire de libération, le Bingtuan va être par la suite la cheville ouvrière de l'implantation des populations han programmée par les autorités centrales. Outre des militaires, cette 
organisation va accueillir des cadres administratifs, des ingénieurs, des ouvriers qualifiés mais aussi des paysans sans terre. L'essor remarquable de la superficie cultivée du Xinjiang qui passe de 1,2 million d'ha. en 1949 à 4,1 millions d'ha. en 2005 est en grande partie dû au Bingtuan qui, par des travaux de bonification et d'irrigation, a permis l'extension d'anciennes oasis, la création de périmètres irrigués modernes et l'installation de plus de 200 fermes lui appartenant. Ce dernier contrôle aussi l'essentiel des territoires pastoraux situés au contact de la frontière du Xinjiang. Mais sa tâche a aussi été de construire des routes, des voies ferrées et des villes nouvelles comme celles de Shihezi ou de Kuytun. Le développement agricole mais aussi urbain et industriel est donc au cœur de cette puissante organisation qui joue aujourd'hui un rôle considérable dans l'économique régionale. Avec une population estimée en 1993 à quelques 2,2 millions de personnes (Pannell et Ma, 1997) le Corps de Production et de Construction du Xinjiang exploiterait en 2007 1,05 million d'ha de terre cultivées et contrôlerait plus de 5000 entreprises industrielles, commerciales et de transport réalisant 37,4 \% des exportations de la région autonome du Xinjiang (Kellner, 2008).

En dépit d'une propagande officielle qui retient l'enthousiasme et le volontarisme de milliers de chinois venus participer au développement de l'Extrême Ouest chinois, ce dernier constitue cependant une terre de relégation. Pendant la Révolution culturelle (1966-1969), l'afflux annuel de migrants forcés a été en moyenne de 100000 personnes avec un maximum de 350000 en 1966. Toutefois, cette population déportée a été autorisée à regagner la Chine de l'Est au début des années 1980. La région compte aussi des «colons spéciaux» dans les «camps de rééducation» (laogai), Han déportés, puis assignés à rester au Xinjiang une fois leur peine purgée.

Parallèlement, s'organise aussi une migration spontanée amorcée à partir de 1958 lors $\mathrm{du}$ «Grand Bond en avant». En raison de son éloignement géographique et de sa moindre politisation, le Xinjiang moins touché que le reste de la Chine, accueille des Han fuyant la famine et les persécutions qui sévissent à l'est. Depuis la fin des années 1980, un nouveau courant migratoire spontané se met en place motivé par les possibilités d'emploi crées par la politique d'ouverture économique inaugurée à partir de 1992 et suivie du programme de développement de l'Ouest chinois ${ }^{4}$ lancé en 2000. L'exploitation des nouveaux gisements d'hydrocarbures, la construction d'oléoduc, de gazoduc et de routes, la mise en chantier de complexes pétrochimiques parmi les plus importants de Chine (Dushanzi et Luntai) ou tout simplement l'essor du commerce sont autant d'opportunités qui attirent les migrants venus des régions densément peuplées du Sichuan, du Hénan et du Jiangxi. Si l'Etat central ne semble plus actuellement organiser la migration, il la stimule cependant tant par l'importance des financements affectés au développement de l'Ouest que par l'attribution gratuite de logement ou la distribution de terre aux migrants Han dans les zones considérées comme prioritaires par les programmes de développement.

16 Au total, la région aurait reçue entre 1947 et 1993 un peu plus 3,7 millions de migrants Han constituant 21,13 \% de la croissance démographique de cette période (Ren, Yuan, 2003). Si les minorités nationales ont une natalité plus vigoureuse que les Han; elles ne sont pas soumises à la règle de l'enfant unique mais bénéficient du fait de leur statut de minorité d'une politique du planning familial plus souple permettant d'avoir deux enfants pour les couples citadins et trois pour les ruraux; la balance ethnique penche cependant de plus en plus en faveur des Han dont l'importance numérique approche, voire dépasse le seuil fatidique des $50 \%$ de la population totale. Au regard des chiffres 
officiels du dernier recensement de 2000, le Xinjiang compterait sur une population de 18,46 millions d'habitants $40,6 \%$ de Han contre 59,4\% pour les minorités nationales ${ }^{5}$. Mais le nombre de Han semble largement sous estimé ${ }^{6}$ dans la mesure où les militaires du Corps de Production et de Construction du Xinjiang ne sont pas comptabilisés dans le recensement au même titre que la population «flottante » des mingong. Les migrants qui viennent travailler temporairement sur les chantiers de construction ou dans les campagnes pour la récolte du coton sont en effet nombreux à rester sur place sans permis de résidence une fois leur contrat terminé.

17 Le résultat de cette politique de colonisation a bouleversé la géographie de ce Grand Ouest. Traditionnellement c'est le sud du Xinjiang(Nanjiang),

c'est-à-dire l'espace occupé par le bassin du Tarim situé au sud de la chaîne des Tian Shan, qui était le plus peuplé. C'était le monde ouïgour populeux des oasis organisées en chapelet autour du désert du Taklamakan, foyer d'une riche agriculture sédentaire et d'une vie urbaine liée aux vieilles cités commerciales des Routes de la soie (Kashgar, Khotan, Aksu, Kutcha). Avec $47 \%$ de la population du Xinjiang, ce Sud est désormais dépassé démographiquement et économiquement par le Nord (Beijiang) organisé autour du bassin de Dzoungarie. Pourtant ce Nord était traditionnellement très faiblement peuplé et urbanisé car occupé par les différents groupes de pasteurs nomades kazakh et mongol au genre de vie extensif. Ce retournement de l'espace où le nord constitue désormais le centre de gravité démographique et économique de la région autonome s'explique par les considérations économiques de la dynamique pionnière. Souffrant moins de l'aridité, les zones pastorales du Nord pouvaient facilement être défrichées et transformées en vastes domaines agricoles producteurs de grains, d'oléagineux et de betteraves sucrières. Par ailleurs la ceinture du bassin Dzoungar constitue la zone historique de la découverte et de l'exploitation du pétrole, notamment avec le site de Maitag (Dushanzi) mis en service dès 1907 (Khamraev, 1998). C'est selon cette logique qu'a été tracée la ligne de chemin de fer du Nord-Xinjiang entraînant selon un axe linéaire la promotion de nouvelles villes chinoises (Urumqi, Shihezi, Kuytun, Karamay) servant d'appui à la diffusion du peuplement han. En 2000, les Han sont majoritaires dans tout le nord à l'exception des préfectures de l'Altaï et de l'Ili où ils constituent autour de $40 \%$ de la population totale. En revanche, le Sud reste en 2000 majoritairement ouiggour y compris dans les villes où les Han ont plutôt tendance à se concentrer. Ainsi à l'exception d'Aksu et Korla, les Han sont minoritaires dans tous les districts urbains du sud: ils représentent $8 \%$ de la population d'Artush, $20,7 \%$ à Kashgar, 16,7 \% à Khotan et 21,3\% à Turpan (BST, 2001). Cependant, depuis 2000, la situation ethnique évolue rapidement car conscient du danger que pouvait constituer un déséquilibre croissant entre un nord sinisé, plus riche, plus urbanisé et industriel face à un sud ouïgour plus pauvre et plus rural, le gouvernement a accentué les efforts d'investissement en direction du sud. Tout le nord du bassin du Tarim est désormais ouvert à la pénétration han depuis l'inauguration en 1999 de la nouvelle voie ferrée du Sud-Xinjiang qui, sur près de $1445 \mathrm{~km}$, relie la ville de Turpan à celle de Kashgar. Les tours de verre de Korla nouvelle capitale pétrolière, l'immense parc industriel et pétrochimique de la ville-chantier de Luntai ou les vastes périmètres irrigués autour d'Aksu témoignent du dynamisme de ce nouveau corridor de développement. Même la marge sud du bassin du Tarim est désormais atteinte par l'élan de la colonisation depuis que l'obstacle du plus grand désert de Chine, le Taklamakan, a été vaincu. Deux grandes routes ouvertes respectivement en 1995 (Luntai/Minfeng) et 2007 (Aksu/ Khotan) traversent du nord au sud sur plus de $500 \mathrm{~km}$ le grand erg (fig. 4). 
Figure 4 - La route stratégique Luntai/Minfeng

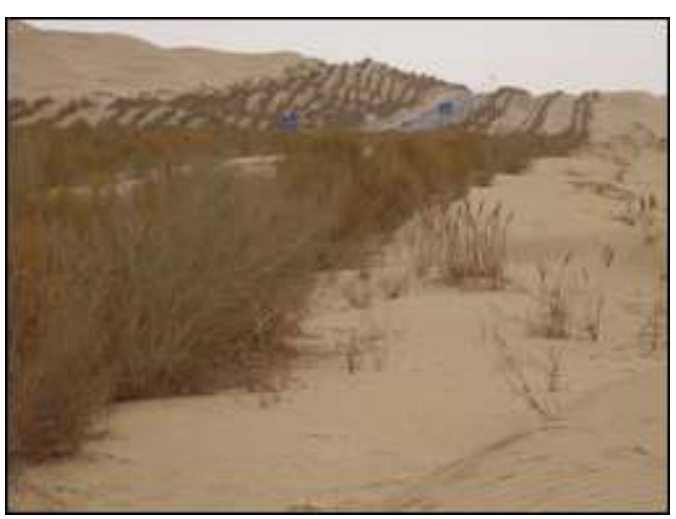

Ouverte à travers le Taklamakan nécessite une protection permanente contre l'ensablement : les dunes sont fixées par des plantation d'arbustes irrigués et des barrières de tige de roseau (A. Cariou, 2008).

Ces grandes pénétrantes permettent d'éviter le long contournement du Taklamakan et de l'ouvrir à la prospection et à l'extraction pétrolière comme pour le gisement de Tazhong situé en plein cœur du désert. Les vieilles cités-oasis du sud s'en trouvent transformées. Kashgar, la capitale historique des Ouigour se contracte progressivement et cède la place à un urbanisme à la "chinoise " associant centres commerciaux et immeubles collectifs standardisés (fig. 5 et 6).

Figures 5 et 6 : La vieille ville ouïgour de Kashgar (Lao Cheng)

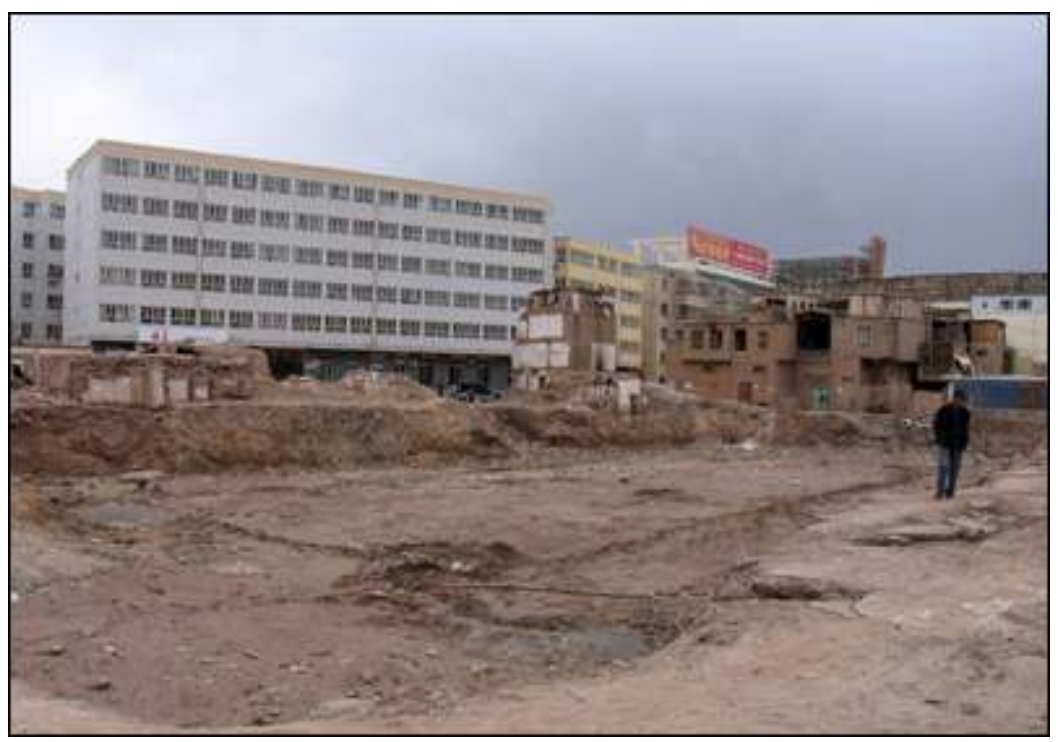




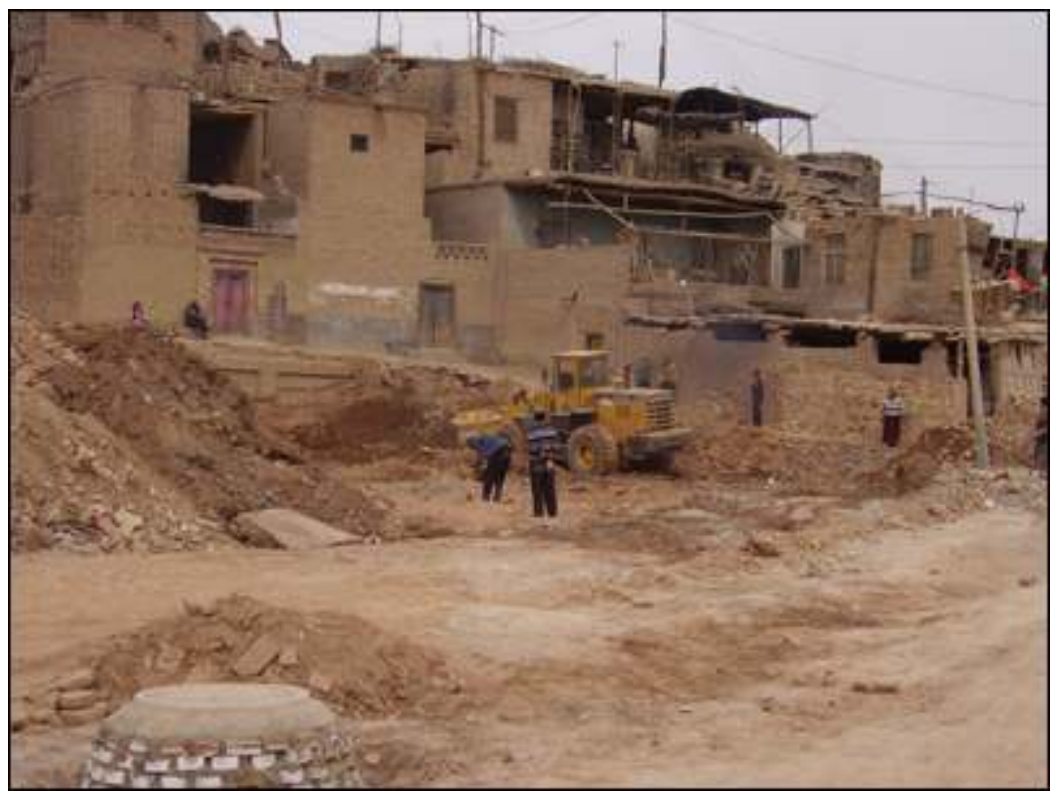

La ville disparaît progressivement sous l'effet de l'expansion d'un modèle urbain chinois fondateur de la ville nouvelle (Xin Cheng). A. Cariou, 2008

Dans l'oasis de Minfeng (Niya), une ville nouvelle encore en chantier se juxtapose à la cité autochtone et accueille déjà les premiers colons. Même dans les campagnes les plus reculées comme dans la petite oasis de Keriya, le mitage du paysage rural traditionnel par des serres traduit la prise de contrôle d'une partie du foncier par des Han.

20 Aujourd'hui, à mesure que la maîtrise du territoire par le pouvoir chinois s'étend de façon systématique à tout le Xinjiang, l'importance démographique et géographique des minorités décline progressivement. Désormais en situation de force, les autorités centrales peuvent alors se consacrer à l'ouverture économique de la région en direction des Etats centrasiatiques.

\section{Le Xinjiang, une nouvelle interface eurasiatique}

\section{L'ouverture sur l'Asie centrale}

Selon un point de vue largement répandu, l'Ouest chinois apparaît comme un véritable «bout du monde » coupé du reste du continent asiatique par l'étendue des déserts et le nœud orographique des hautes chaînes des Kunlun, du Pamir, des Tien Shan et de l'Altaï. Toutefois, en dépit des contraintes topographiques et climatiques, le Xinjiang ne peut être considéré comme un angle mort géographique mais constitue bien au contraire une terre de passage et d'échange entre la Chine, l'Asie centrale et l'Europe et cela dès la plus haute antiquité. Les vestiges des cités-oasis et les tracés des multiples pistes caravanières témoignent d'un Age d'or commercial des Routes de la soie aujourd'hui révolu (Boulnois, 2001). C'est principalement par le couloir naturel et historique de la Porte de Dzoungarie encadrée par les massifs des Tien Shan et de l'Altaï que se sont effectuées les grandes migrations des peuples nomades entre steppes centrasiatiques et Empire chinois. Cependant on sait que cette région a aussi constituée un des espaces les plus fermés du monde du temps de la Guerre froide. La zone frontalière sino-soviétique comptait parmi les plus sensibles et les plus surveillées, 
soumise à un contrôle militaire et à une réglementation spéciale parfois appliquée sur une profondeur de plus de $100 \mathrm{~km}$.

Mais aujourd'hui, avec l'évolution du contexte géopolitique régional le Xinjiang retrouve progressivement son rôle géostratégique d'interface eurasiatique car la disparition de l'URSS en 1991 a été l'occasion pour la Chine d'étendre son influence sur l'Asie centrale. En effet, le chaos économique et politique post-soviétique qui affecte les nouvelles républiques centrasiatiques sert les intérêts chinois. Les jeunes Etats tentent actuellement de rompre avec l'isolement dans lequel ils ont été durablement confinés par l'ancienne puissance tutélaire russe. Leur développement est handicapé par une organisation territoriale marquée par l'enclavement et le poids de l'héritage soviétique: voies de chemin de fer, routes, lignes aériennes, gazoduc et oléoduc convergent encore pour l'essentiel vers le territoire russe. Or la volonté d'indépendance économique et politique à l'égard de Moscou pousse les nouveaux Etats à chercher d'autres partenaires sur la scène internationale. C'est pourquoi la Chine a pris l'initiative de fonder en 1996 "le Groupe de Shanghai", organisation de coopération centrasiatique de sécurité dont le but est de combattre le terrorisme et de limiter la militarisation des zones frontalières. Ce groupe constitué par la Chine, le Kazakhstan, le Kirghizstan, l'Ouzbékistan, la Russie et le Tadjikistan (plus l'Inde, l'Iran, la Mongolie et le Pakistan qui ont le statut d'observateur) s'est restructuré en 2001 pour devenir l'Organisation de Coopération de Shanghai (OCS) et afficher de nouveaux objectifs : renforcer les économies de la région par le développement des transports, des échanges commerciaux et des investissements mais aussi coordonner et assurer la sécurité énergétique. Cette forme d'intégration régionale se substitue d'une certaine manière à la CEI (Communauté des Etats Indépendants), structure voulue par la Russie mais restée inopérante du fait de la volonté centrifuge des "républiques sœurs », et a également pour but de contrer la pénétration des Etats-Unis dans la région (Niquet, 2006).

La Chine se positionne donc comme une alternative à l'enclavement et a l'isolationnisme centrasiatique, le Xinjiang constituant une pièce maîtresse sa stratégie. Ainsi, avec $5600 \mathrm{~km}$ de frontière internationale partagés avec huit Etats riverains (Mongolie, Russie, Kazakhstan, Kirghizstan, Tadjikistan, Afghanistan, Pakistan et Inde) la région autonome apparaît comme un "poste avancé» continental, espace stratégique de première importance pour la diffusion de l'influence chinoise au cœur de l'Asie.

L'ouverture de la Chine sur son hinterland continental s'est traduite depuis les années 1990 par la construction d'un réseau de transport et de communication transfrontalier permettant la connexion avec les Etats voisins. C'est tout naturellement sur l'axe historique de la Porte de Dzoungarie que se sont portés les premiers efforts avec l'aménagement du port sec transfrontalier d'Alatau (Alashankou) côté chinois/Dostyk côté kazakh. Ce point de passage majeur est desservi par un grand axe autoroutier mais aussi ferroviaire ouvrant les républiques d'Asie centrale et la Russie aux produits chinois. Le raccordement ferroviaire entre la Chine et l'URSS prévu dès les années 1950 mais abandonné en 1961 suite au refroidissement entre les deux puissances est achevé depuis 1991. Après le Transsibérien, cette ligne est présentée par les autorités chinoises comme le second "pont ferroviaire " transcontinental entre le port de Lianyungang situé sur la façade pacifique et Rotterdam (Xinhua, 2001). Cette ligne longue de 10900 $\mathrm{km}$ a l'avantage d'être plus courte d'environ $2000 \mathrm{~km}$ que le Transsibérien et permet au train de joindre la Chine littorale à l'Europe de l'Ouest en 8 à 9 jours là ou le bateau met 
un mois. Outre le gain de temps, cette ligne transasiatique permettrait de résoudre en partie le problème de la saturation des ports Chinois et celui de l'enclavement des républiques ex-soviétiques d'Asie centrale avec l'ouverture sur l'océan Pacifique. Même si la Deutsche Bahn a fait circuler en 2008 des wagons de marchandises entre Hambourg et Pékin le succès d'une telle ligne terrestre n'est pas pour le moment probant tant les obstacles sont encore nombreux : multiplicité des acteurs et des franchissements de frontière (Kazakhstan, Russie, Europe de l'Est), problèmes techniques, rupture de charge... (Beyer, 2008) ${ }^{7}$. Enfin s'est par cette Porte de Dzoungarie que transite désormais le pétrole d'Asie centrale en direction de la Chine grâce à la construction d'un oléoduc entre la station d'Atasu au Kazakhstan central et le complexe pétrochimique de Dushanzi au Xinjiang. A l'horizon 2011, un oléoduc de plus de $3000 \mathrm{~km}$ et d'une capacité de 20 millions de tonnes réalisé par les chinois devrait relier la Caspienne au Xinjiang afin d'assurer l'approvisionnement énergétique de la Chine littorale (Peyrouse, 2008).

Fort du succès rencontré par ce port sec qui a traité plus de 15 millions de tonnes de marchandises en 2007, la Chine et le Kazakhstan ont multiplié depuis 1992 les points de passage sur la frontière sino-kazakh et ont raccordé et unifié leur réseau routier par la réfection et la création de routes. Depuis 2008, sept autoroutes sont en construction afin de relier les grands centres urbains situés de part et d'autre de la frontière, via notamment les principaux points de passage de Khorgas, d'Alatau et de Jeiminay.

Si l'ouverture chinoise sur le Kazakhstan est pleinement réalisée, en revanche les points de passage avec les autres pays voisins sont pour le moment relativement limités en dépit de nombreux projets qui prévoient une modernisation des infrastructures existantes. Les liens avec le Kirghizstan se résument à deux points de passage frontaliers, le col de Torugart $(3752 \mathrm{~m})$ qui mène à Bichkek via Narin et le poste d'Irkeshtam qui conduit vers Och et la vallée du Ferghana. Un seul poste de transit assure le lien avec le Tadjikistan par le col de Kulma (4 $363 \mathrm{~m}$ ) ouvert seulement aux commerçants chinois et tadjiks de mai à septembre ce qui permet de désenclaver le plateau du Pamir et le Badakhshan désormais plus polarisés par Kashgar que par Douchambé la capitale tadjike. Si la Chine a réalisé de bonnes routes asphaltées jusqu'à ses frontières, en revanche se sont des pistes qui pour le moment prennent le relais côté kirghiz, tadjik et mongol (poste de Laoyemiao) ce qui limite fortement les échanges.

Enfin faut-il signaler l'importance de la grande route du Karakoram reliant Kashgar à Islamabad au Pakistan via le col de Kunjerab $(4655 \mathrm{~m}$ ). Longue de $800 \mathrm{~km}$ cette route considérée comme la plus haute du monde a été achevée en 1978 mais n'a été ouverte au commerce qu'en $1982^{8}$ (Kreutzman, 1991). Cette route est avant tout stratégique plus que commerciale car elle permet de contrôler la zone frontalière sensible et montagneuse que se partagent la Chine, le Pakistan, l'Inde et l'Afghanistan. D'ailleurs sa création résulte du contexte géopolitique des années 1970 où l'alliance sinopakistanaise était une réponse à la coopération Inde-URSS.

Désormais au centre d'un nouveau réseau routier et ferroviaire le Xinjiang constitue une « tête de pont » commerciale entre la Chine et l'Asie centrale. 


\section{L'ouverture économique et le développement frontalier}

29 Afin d'assurer le succès de cette nouvelle «fenêtre " continentale, le gouvernement chinois ne s'est pas contenté d'améliorer les infrastructures de transport et de multiplier les points de passages frontaliers mais accompagne sa politique d'ouverture par l'aménagement de toute une série de "zones économiques spéciales » destinée à stimuler le commerce international tout en assurant un développement productif de l'Ouest chinois à partir d'investissements directs nationaux et étrangers. Cette politique d'ouverture fait suite au succès des premiers échanges transfrontaliers informels amorcés à la fin des années 1980. Lors du dégel des relations sino-soviétiques, les citoyens frontaliers des deux pays avaient reçu l'autorisation de circuler sans visa ce qui a permis l'essor du commerce de valise pratiqué par les minorités nationales. Fins connaisseurs des marchés locaux et des réseaux de solidarité, Ouïgour, Kazakh, Kirghiz et Doungane ont alors alimenté des flux transfrontaliers de biens de consommation courantes chinois en direction de l'URSS puis des républiques ex-soviétiques frappées par le déclin des échanges avec Moscou. Ce commerce informel florissant a permis à certaines familles de s'enrichir considérablement. Conscientes de l'importance de l'enjeu économique, les autorités chinoises ont décidé de structurer, d'amplifier et de reprendre en main cette source de profit ce qui s'est traduit par l'éviction progressive des minorités nationales au profit des commerçants han.

30 Ainsi, à l'instar des villes côtières de la Chine, les villes de Yining, Bole et Tacheng, frontalières du Kazakhstan, mais aussi Urumqi sont dotées depuis 1992 du statut de « ville frontalière ouverte » où des mesures fiscales avantageuses et l'aménagement de zones de coopération économique frontalières sont destinées à attirent les investisseurs. En suivant la même logique des zones franches développées dans les villes de Chine littorale, Pékin inaugure en 2003 la première zone franche frontalière de Chine à Khorgas sur la frontière avec le Kazakhstan. Outre l'installation de plusieurs centaines de sociétés spécialisées dans les produits manufacturés et les nouvelles technologies, la zone franche accueille sur plus de $15 \mathrm{~km}^{2}$ toutes les infrastructures nécessaires à ce nouveau commerce international: services bancaires, logistiques, hôtels, halls d'exposition pour les produits chinois. En 2007, plus de trois millions de personnes et deux millions de tonnes de marchandises auraient transité par ce poste de Khorgas (Peyrouse, 2008). Fort de ce succès, il existe aujourd'hui plus de 16 ports secs dotés de zones commerciales frontalières, de fonction d'entreposage, de services logistiques à l'image de la zone franche de Jeiminay ouverte en 2006 sur plus de 100 hectares et destinée à rayonner sur le nord du Kazakhstan et la Russie (Xinhua, 2006).

31 Afin d'augmenter le volume des biens exportables et de réduire le retard économique du Xinjiang, le gouvernement encourage l'aménagement de «zones de développement économique et technologique» au régime fiscal attractif. Ce statut de «zone économique spéciale » conféré aux villes du bassin Dzoungar à partir de 1992 (Urumqi, Shihezi et Kuitun) a été plus récemment étendu à celles de Korla et Luntai situées dans le bassin du Tarim (fig. 7). 
Figure 7 - Kola, nouvelle capitale pétrolière du bassin du Tarim

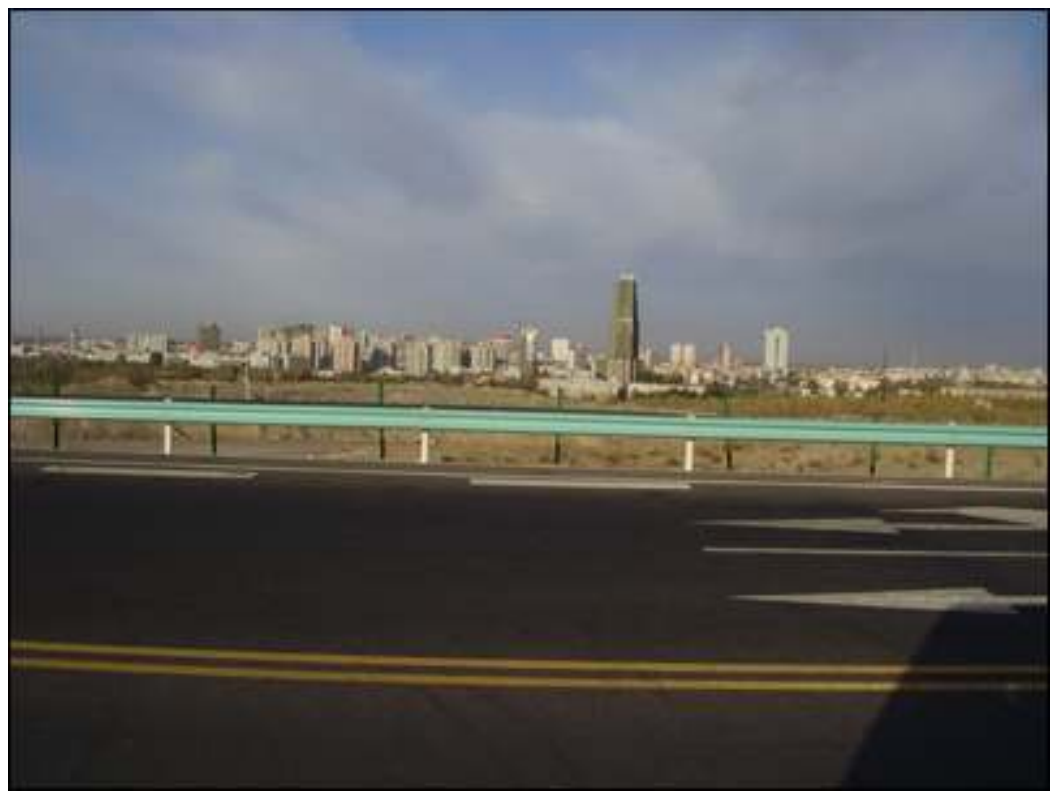

Symbole de la réussite financière, industrielle et technologique aux portes du désert du Taklamakan (A. Cariou 2006).

De vastes superficies de plusieurs $\mathrm{km}^{2}$ gagnées sur la steppe accueillent désormais des industries légères et lourdes qui transforment pour l'essentiel les matières premières présentes sur place: industries alimentaires (jus de fruit, bière, sauce tomate), industries chimiques (engrais, matières plastiques), industries textiles (coton, cuir).

En définitive, la désorganisation et l'effondrement des flux économiques en Asie centrale suite à la disparition de l'URSS ont été les signes annonciateurs d'une montée en puissance de la Chine dans la région ce dont témoignent les termes de l'échange. Ainsi, cette dernière exporte essentiellement vers l'Asie centrale des produits finis de consommation courante (produits agroalimentaires et pharmaceutiques, textile, chaussures, pièces détachées, électroménager, électronique) tandis qu'elle importe principalement des matières premières brutes : pétrole, métaux ferreux et non ferreux, laine, cuir. Grâce à la prospection et à l'acquisition de gisements d'hydrocarbures au Kazakhstan et à la réalisation de gazoducs et d'oléoducs, les sociétés chinoises investissent le terrain autrefois chasse gardée des Russes. En visant à court terme l'approvisionnement du pays à partir du gaz ouzbek et turkmène, du pétrole kazakh et russe qui viendront s'ajouter aux hydrocarbures du Xinjiang, la Chine entend faire de l'Asie centrale un pôle majeur de sa stratégie énergétique (Pannell et Schmidt, 2006).

Au total, le Xinjiang s'affirme aujourd'hui comme une zone dynamique du commerce frontalier notamment avec le Kazakhstan, pays le plus riche de l'espace post-soviétique derrière la Russie, qui s'impose comme une plate-forme de consommation et de redistribution des produits chinois vers les autres républiques d'Asie centrale et la Russie. Le commerce sino-kazakhstanais qui constitue $70 \%$ de l'ensemble du commerce sino-centrasiatique a connu un essor phénoménal au cours des dernières décennies : en 1990, le volume du commerce frontalier entre le Xinjiang et le Kazakhstan était de 1 milliard de dollars, il est passé à 12 milliards de dollars en 2007. Selon les experts locaux, l'impact commercial de la Chine dans ce pays aurait relégué la Russie au second rang (Peyrouse, 2008). 


\section{Conclusion} l'implantation chinoise incapable de s'inscrire durablement dans la géographie d'une périphérie qui lui a été toujours culturellement différente et hostile. Au regard de ce constat, les dernières décennies apparaissent comme une rupture et une charnière. Alors que la région autonome est désormais solidement arrimée au reste du pays par le développement d'infrastructures de transport et de communication et que le désenclavement intra-régional est pratiquement achevé, plus rien ne semble désormais s'opposer à l'implantation han massive et à l'assimilation programmée des minorités nationales, celles-ci étant en passe d'être minoritaires sur leur propre territoire. Le processus en cours sera-t-il comparable à celui vécu par la Région autonome de Mongolie intérieure où les Mongol très largement sinisés ne représentent plus que $14 \%$ de la population contre $82 \%$ de Han ? Quoi qu'il en soit, le Xinjiang est plus que jamais solidement intégré au reste du territoire chinois. Avec le programme d'investissement pour le Grand Ouest, une course au développement est engagée afin de réduire le gradient de périphéricité : certes les activités agricoles, minières et énergétiques prédominent mais des efforts importants d'industrialisation et de tertiarisation de l'économie ont été réalisés, notamment avec l'ouverture commerciale sur l'Asie centrale. Le gouvernement chinois mise sur l'élévation du niveau de vie et le développement économique du Xinjiang afin de le stabiliser et de mieux faire accepter la sinisation auprès des minorités nationales. Pourtant celles-ci restent faiblement engagées dans l'économie moderne tant en raison de leur relégation par les Han que par leur attitude volontairement conservatrice, la tradition et le refus de la modernisation constituant un rempart contre l'assimilation. A l'échelle du Xinjiang, l'accentuation des écarts de développement socioéconomiques entre Han et minorités nationales constitue un des défis majeurs, à l'image des écarts de développement régionaux qui fragilisent et risquent de déstabiliser la Chine toute entière.

\section{BIBLIOGRAPHIE}

Aubin F., 1990, Donneurs ou preneurs, quelques réflexions sur le jeu des transferts culturels entre Chinois, Turcs et Mongols, in Dor R. L'Asie centrale et ses voisins, Inalco, Paris, p. 165-180.

Beyer A., 2009, Le contact des réseaux ferrés à l'écartement russe et européen. Héritage embarrassant ou futur trait d'union continental ? States, $\mathrm{n}^{\circ} 14$ (à paraître).

Boulnois L., 2001, La Route de la Soie. Dieux, Guerriers et Marchands, Editions Olizane, Genève, 558 p. BST (Bureau des statistiques du Xinjiang), 2001, Xinjiang tongji nianjian, Beijing, China Statistics Press.

Hopkirk P., 1995, Bouddhas et rôdeurs sur la route de la Soie, Editions Picquier, Arles, 345 p.

EchoGéo, 9 | 2009 
Jan M., 1998, L'intégration du Xinjiang dans l'ensemble chinois. Vulnérabilité et sécurité. Cahiers d'étude sur la Méditerranée orientale et le monde turco-iranien, n 25, p. 65-82.

Khamraev H., 1998, La géopolitique du pétrole, Cahiers d'étude sur la Méditerranée orientale et le monde turco-iranien, $\mathrm{n}^{\circ} 25$, p. 139-149.

Kellner T., 2008, L'Occident de la Chine. Pékin et la nouvelle Asie centrale (1991-2001), Presses Universitaires de France, Paris, 622 p.

Kreutzman H., 1991, The Karakoram Highway : The Impact of Road Construction on Mountain Societies. Modern Asian Studies, vol. 25, n 4, p. 711-736.

Kreutzman H., 1997, Minderheiten und Mehrheiten in Xinjiang : Auswirkungen staatlicher Nationalitätenpolitik im Nordwesten Chinas. Geographische Rundschau, vol. 49, n 4, p. 711-736.

Kumul A., 1998, Le « séparatisme ouïgour au XX ${ }^{\mathrm{e}}$ siècle : histoire et actualité. Cahiers d'étude sur la Méditerranée orientale et le monde turco-iranien, $\mathrm{n}^{\circ}$ 5, p. 266-271.

Lothaire F, 2006, La Chine et ses minorités : les Ouïghours entre incorporation et répression, L'Harmattan, Paris, 196 p.

Niquet V., 2006, La Chine en Asie centrale. Perspectives chinoises, n 96, p. 2-10.

Pannell C., Ma L., 1997, Urban Transition and Interstate Relations in a Dynamic Post-Soviet Borderland : The Xinjiang Uygur Autonomous Region of China. Post-SovietGeography and Economics, vol. 38, n 4, p. 206-229.

Pannell C., Schmidt P., 2006, Structural Change and Regional Disparities in Xinjiang, China. Eurasian Geography and Economics, vol. 47, n 3, p. 329-352.

Patterson Giersch C., 2008, « Le deuil du Tibet ». La conception de l'Etat moderne dans l'Asie intérieur de la fin des Qing.Perspectives chinoises, $\mathrm{n}^{\circ}$ 3, p. 4-35.

Peyrouse S., 2007, The Economic Aspects of the Chinese Central-Asia Rapprochement. Silk Road Papers, The Central Asia and Caucasus Institute, 74 p. http://www.isdp.eu/files/publications/srp/ 07/sp07economicaspects.pdf

Peyrouse S., 2008, La présence économique chinoise au Kazakhstan. Volontarisme chinois et appréhension centre-asiatiques. Perspectives chinoises, $n^{\circ}$ 3, p. 36-52.

Ren Q., Yuan X., 2003, Impacts of Migration to Xinjiang Since the 1950s, China's Minorities on the Move : Selected Case Studies, Edited by Robyn Iredale, Naran Bilik and Fei Guo, New York, p. 89-105. Renard J.-P., Picouet P., 1993, Frontières et territoires, La documentation Française, Paris, 44 p. Sellier J. et Sellier A, 2007, Atlas des peuples d'Orient. Moyen-Orient, Caucase, Asie centrale, La Découverte, Paris, 206 p.

Simonet L., 2007, Oléoducs et gazoducs chinois à l'aube du XXI ${ }^{\mathrm{e}}$ siècle : les vecteurs d'une diplomatie de l'énergie. Monde Chinois, $n^{\circ}$ 9, p. 25-37.

Toops S., 1992, Recent Uygur Leaders in Xinjiang. Central Asia Survey, vol. 11, n² 2, p. 86-101.

Wang D., 1995, Xinjiang of the 20th Century in Historiography. Central Asia Survey, vol. 14, $\mathrm{n}^{\circ} 2$, p. 265-283.

Xinhua, 2008, Le quotidien du peuple en ligne, http://french.peopledaily.com.cn/Economie/ 3722221.htm 


\section{NOTES}

1. Le discours officiel chinois considère généralement avec une certaine condescendance les minorités ethniques du fait de leur différences sociologiques, religieuses et linguistiques.

2. Les Han constituent les Chinois ethniques qui se distinguent au Xinjiang des minorités nonhan constituées par les peuples turciques (Ouigour, Kazakh, Kirghiz), mongol et persan (Tadjik).

3. En Asie centrale soviétique, la disparition de l'URSS a permis l'émergence de cinq républiques indépendantes : Kazakhstan, Kirghizstan, Ouzbékistan, Tadjikistan et Turkménistan.

4. L'Ouest chinois correspond à un ensemble géographique défini par le pouvoir central qui regroupe douze entités administratives couvrant environ $60 \%$ du territoire chinois et concernant $30 \%$ de la population du pays. Outre le Xinjiang, on y trouve le Gansu, le Guangxi, le Guizhou, la Mongolie intérieure, le Ningxia, le Qinghai, le Shaanxi, le Sichuan, le Yunnan, le Tibet et la municipalité de Chongqing.

5. En 2000, les minorités au Xinjiang se composent comme suit : 45,2\% de Ouigour, $6,74 \%$ de Kazakh, 4,55 \% de Hui, 0,86 \% de Kirghiz, 0,81 \% de Mongol, 0,30 \% de Dongxiang, 0,21 \% de Tadjik. 6. Les chiffres officiels du recensement sont à prendre avec une certaine prudence. Beaucoup d'observateurs estiment que l'importance numérique des Han du Xinjiang serait volontairement minorée afin d'éviter le renforcement de l'irrédentisme ouïgour.

7. Le réseau ferré chinois et d'Europe de l'Ouest et un réseau à "voie normale " avec un écartement de $1435 \mathrm{~mm}$ tandis que le réseau de l'ex-Union soviétique et de ses ex-pays satellites est à « voie large » avec un écartement de $1520 \mathrm{~mm}$. Cette différence d'écartement constitue un obstacle à la fluidité du trafic.

8. En raison de l'altitude, le trafic commercial transfrontalier y est interrompu du 30 novembre au $1^{\mathrm{er}}$ mai.

\section{RÉSUMÉS}

Le Xinjiang est généralement présenté comme l'archétype de la périphérie chinoise en raison de son enclavement, de son retard de développement et de son peuplement constitué de minorités nationales. Pourtant, depuis peu, cette périphérie présente le paradoxe d'un développement accéléré qui la singularise des autres provinces intérieures de la Chine par son premier rand pour son IDH et son PIB. Cette évolution s'explique par son rôle géostratégique dû à sa richesse en ressources naturelles et à sa position frontalière ce qui lui confère la fonction de "tête de pont " commerciale de la Chine en Asie centrale. L'achèvement du réseau routier et ferroviaire transcontinental porteur de migrations Han participe de la politique nationale de sécurisation et d'intégration économique et culturelle du Xinjiang ce que traduit le rapide déclin des minorités nationales.

This paper analyses recent geographic change in the Xinjiang Uygur Autonomous Region. Xinjiang plays a geostratégic role in China due to abundance of natural resources such as oil, gas an coal but also for its particular geographic location in the heartland of Central Asia. The area gives to China an high potentiel for trade with the newly independent Central Asian republics and Eurasia. That's the reason why central government take active measures for an acceleration of the economic development in the region with the aim of promoting regional security, cultural and économic integration. Nowadays, an unprecedent change taken place in Xinjiang with the 
improvements in transportation infrastructure. The expansion of the transcontinantal railway and highways between Est China to Central Asia has created a fast growing economies and the increase of Han migration. The rapide decline of the proportion of ethnic minority nationality is the result of a political strategy of Chinese national expansion and territorial intergation in order to control the Xinjiang.

\section{INDEX}

Keywords : Xinjiang, China, Central Asia, East Turkestan, regional development and intégration Mots-clés : Xinjiang, Chine, Asie centrale, Turkestan oriental, intégration et développement régional

\section{AUTEUR}

\section{ALAIN CARIOU}

Alain Cariou (alain.cariou@wanadoo.fr) est Maître de conférences à l'Université Paris IVSorbonne et membre de l'UMR Prodig. Il a notamment publié :

- L'Asie centrale dans la mondialisation : de la marginalisation à l'intégration, in Cadène P., La mondialisation. L'intégration des pays en développement, SEDES, Paris, 2007, p. 163-174.

- Ouzbékistan : le paradoxe de l'enracinement rural, Espace, Populations, Sociétés, n 1, 2007, p. 101-112.

- Entre repli et ouverture : nouveaux enjeux et recompositions des déserts centrasiatiques, Maghreb, Machrek, n 193, 2007, p. 109-131. 